\title{
Experimental and computational studies of the interactions between carbon nanotubes and ionic liquids used for detection of acetaminophen
}

\author{
Michele A. Salvador ${ }^{\mathrm{a}, *}$, Camila P. Sousa ${ }^{\mathrm{b}}$, Cleiton D. Maciel ${ }^{\mathrm{a}, \mathrm{c}}$, Rayane N. Gomes ${ }^{\mathrm{b}}$, \\ Simone Morais ${ }^{\mathrm{d}}$, Pedro de Lima-Neto ${ }^{\mathrm{b}}$, Maurício D. Coutinho-Neto ${ }^{\mathrm{a}}$, Adriana N. Correia ${ }^{\mathrm{b}}$, \\ Paula Homem-de-Mello, \\ a Centro de Ciências Naturais e Humanas, Universidade Federal do ABC, Av. dos Estados, 5001, Bloco B, sala 1017, 09210-580, Santo André, SP, Brazil

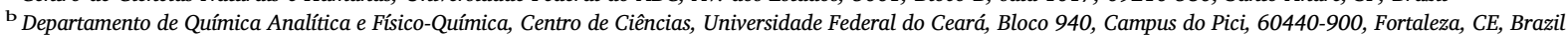 \\ ${ }^{\mathrm{c}}$ Instituto Federal de Educação, Ciência e Tecnologia de São Paulo, Campus Itaquaquecetuba, Avenida Primeiro de Maio, 500, 08571-050, Itaquaquecetuba, SP, Brazil \\ d Instituto Superior de Engenharia do Porto, Instituto Politécnico do Porto, Rua Dr. Bernardino de Almeida 431, 4200-072, Porto, Portugal
}

\author{
Keywords: \\ Paracetamol \\ 1-Butyl-3-methylimidazolium \\ hexafluorophosphate \\ MWCNTs \\ Density Functional Theory \\ Molecular dynamics \\ Monte Carlo
}

\begin{abstract}
A B S T R A C T
The interactions between multi-walled carbon nanotubes and different amounts of an ionic liquid (IL), as well as the interactions between this system (used as electrochemical sensor) and acetaminophen (ACOP), were investigated through both experimental and theoretical methodologies. Experiments indicated that there is an optimal concentration of ionic liquid for ACOP detection. A host of techniques and model systems were employed to investigate the adsorption and oxidation processes. To investigate the source of the increased electrochemical current in the presence of an IL, we computed the adsorption energy values of ACOP in the nanotube - IL system via Monte Carlo simulations and Density Functional Theory (DFT). DFT allowed us to explore the changes in adsorption energy due to oxidation. Our theoretical results support the experimental findings that moderate amounts of IL modulates ACOP/ACOP ${ }^{+}$adsorption, pointing to a cooperative effect that tends to wane with increasing amounts of IL pairs. We observed that the IL favors desorption of the oxidized species and facilitates charge transfer from the ACOP to the nanotube. Therefore, our studies point towards multifactorial effects with clear physical basis that modulates binding leading to an optimal ratio to promote ACOP detection.
\end{abstract}

\section{Introduction}

Carbon-based materials such as graphite [1-4], graphene [5-12] and carbon nanotubes $[8,12,13]$ have been widely used as electrodes for electrochemical sensing. These materials present such advantages as high electroactive areas, high electrical conductivities, high reactivity and chemical stability [14]. Furthermore, the materials' properties can be improved by functionalization with different materials, such as metallic nanoparticles [13], surfactants [15] and different types of polymers [16]. However, ionic liquids [17] present unique characteristics such as high boiling points and conductivities. In combination carbon nanotubes and IL may be suitable materials in the development of detectors for different compounds as they can have synergistic effects such as hydrophobic interactions, $\pi-\pi$ bonds, electrostatic interactions, and hydrogen bonds [18].

In fact, the interactions among these carbon structures and ionic liquids have been extensively studied by different experimental [19-21] and computational methodologies [22-26]. García et al. [27] studied the interaction between ionic liquids, nanotubes and graphene sheets via molecular dynamics and quantum calculations, and analyzed both adsorption and confinement phenomena. The molecular dynamics results showed a strongly structured fluid characterized by anion-cation hydrogen bonding, and especially by anion-anion hydrogen bonding. Quantum calculations showed that the interaction between IL and carbon nanostructures had no dramatic effect on the electronic structure of the graphene or carbon nanotube, and van der Waals interactions were the driving force for the adsorption on the surface. Alarcón et al. [28] investigated the structure and orientation of water molecules at model hydrophobic surfaces. Graphene sheets, carbon nanotubes and fullerenes with different radii were modeled to consider the role of curvature of the carbon structures in their hydration process. For the graphene sheet and the larger radii nanotubes, the water molecules closer to the surface were found to be more structured and oriented than the bulk, while for nanotubes and fullerenes with sub-nanometric diameters, this structuring and orientation is quickly lost because of the geometrical constraints imposed by the surface.

\footnotetext{
* Corresponding authors.

E-mail addresses: michelesalvador@gmail.com (M.A. Salvador), paula.mello@ufabc.edu.br (P. Homem-de-Mello).
} 
Previously, we proposed a sensor composed of multi-walled nanotubes dispersed in polyethylenimine for the electrochemical detection of a popular pharmaceutical drug used worldwide: acetaminophen (ACOP) or paracetamol [28]. In addition to establishing an analytical tool for drug detection, we showed that $\pi-\pi$ interactions drive the detection of ACOP by nanotubes.

In this study, we employed experimental techniques to evaluate the influence of different amounts of ionic liquid on the electrochemical detection of ACOP. Theoretical model calculations are used to provide an atomistic description of the observed phenomena and to offer possible explanations to the marked changes in current observed.

\section{Methodology}

\subsection{Experimental materials and procedures}

Acetaminophen (ACOP), 1-Butyl-3-methylimidazolium hexafluorophosphate $\left([\mathrm{BMIM}]^{+}\left[\mathrm{PF}_{6}\right]^{-}\right)$, Nafion ${ }^{\circ}\left(10 \mathrm{wt} \%\right.$ in $\left.\mathrm{H}_{2} \mathrm{O}\right)$, and multi-walled carbon nanotubes powder (MWCNT, 6-9 nm diameter, 5 microns length) were acquired from Sigma Aldrich. The BR buffer solution was made up of phosphoric acid, boric acid, and acetic acid, and the $\mathrm{pH}$ value was adjusted with $\mathrm{NaOH}$. All reagents used in this study were analytical grade. All the solutions were prepared with water purified by a Milli Q System (Millipore Corp.). The ACOP standard solution was prepared by dissolving an appropriate quantity of ACOP in ultrapure water. This solution was later stored in a dark flask and kept in a refrigerator to prevent degradation.

Electrochemical measurements were performed on an Autolab PGSTAT 101 Metrohn- Eco Chemie controlled by a personal computer, using Nova version 1.11.2 software. A conventional three-electrode system, which was composed of a bare or modified glassy carbon electrode (GCE) (BASi, $3 \mathrm{~mm}$ diameter) as the working electrode, a Pt sheet as the auxiliary electrode and an $\mathrm{Ag}_{(\mathrm{s})} / \mathrm{AgCl}_{(\mathrm{s})} / \mathrm{Cl}^{-}$(aq) (saturated $\mathrm{KCl})$ as the reference electrode. When it was necessary, a Microanal B474 $\mathrm{pH}$ meter equipped with a $3.0 \mathrm{~mol} \mathrm{~L}{ }^{-1} \mathrm{Ag} / \mathrm{AgCl} / \mathrm{Cl}^{-}$glass combined electrode was used to adjust the $\mathrm{pH}$ values by adding appropriate amounts of $1.0 \mathrm{~mol} \mathrm{~L}{ }^{-1} \mathrm{NaOH}$ stock solution. The electrodes were cleaned in a Quimis model Q335D ultrasonic cleaner equipped with a heating bath.

GCE was polished with diamond paste to a grain size $3 \mu \mathrm{m}$, washed with ultrapure water, immersed in ethanol and placed in an ultrasound bath for $5 \mathrm{~min}$. This immersion/sonication procedure was repeated with purified water. Finally, the surface of the GCE was dried with a stream of $\mathrm{N}_{2}$ gas.

The MWCNTs were functionalized by acid treatment with concentrated sulfuric acid and nitric acid (3:1 v/v) [29,30]. This mixture was moderately stirred with a magnetic stir bar for $12 \mathrm{~h}$ at $26 \pm 1{ }^{\circ} \mathrm{C}$. Next, the suspension was vacuum filtered, washed several times with ultrapure water until a $\mathrm{pH}$ of 7.0 was achieved, and dried at $70^{\circ} \mathrm{C}$ for $12 \mathrm{~h}$. This strong acid treatment promotes the generation of functional groups such as carboxyl, hydroxyl, quinone, nitro, and amino groups, on the surface of the MWCNTs [31,32]. The functionalized multi-walled carbon nanotubes are represented along this text by $f$-MWCNT. The $f$ MWCNT - IL was obtained by mixing different amounts of $f$-MWCNTs and IL in weight with $0.5 \% \mathrm{w} / \mathrm{w}$ of Nafion ${ }^{\circ}$ in dimethylformamide. Several ratios of $f$-MWCNTs and IL were evaluated, including 1.0:0.0; 1.0:1.0; 1.0:2.0; 1.0:5.0; 1.0:10.0; 1.0:20.0 and 0.0:1.0 (w/w).

To prepare the modified GC electrode a drop $(1.5 \mu \mathrm{L})$ of the resulting dispersion was cast onto the GCE surface using a $5 \mu$ syringe to prepare the IL/f-MWCNTs/GC electrode. The electrode was then dried at approximately $60^{\circ} \mathrm{C}$ for $15 \mathrm{~min}$ in an oven lamp, which was made in the laboratory. When not in use the modified electrode was stored in a desiccator at room temperature.

An electrochemical cell containing $10.0 \mathrm{ml}$ of supporting electrolyte was used for the electrochemical experiments. Between each experiment, the solution was stirred using a stirrer for three minutes, to ensure the reproducibility across all experiments. Square wave voltammetry (SWV) was used to assess the electrooxidation of the ACOP excluding any adsorption by the modified electrode. SWV was the electrochemical technique performed to detect and quantify the ACOP [33].

\subsection{Computational details}

Molecular dynamics simulations of paracetamol (ACOP) in water and in $[\mathrm{BMIM}]^{+}\left[\mathrm{PF}_{6}\right]^{-}$were performed using the Gromacs package [34], which was intended to investigate the role of specific intermolecular interactions with different solvents. The simulation of ACOP in water was performed with parameters based on an OPLS-AA force field [34], in the isothermal-isobaric ensemble with standard conditions for temperature and pressure $(\mathrm{T}=298 \mathrm{~K}$ and $\mathrm{P}=1 \mathrm{~atm})$. The system was formed by one ACOP molecule immersed in 883 water molecules inside a cubic box, with periodic boundary conditions and minimum image convention [35,36]. The SPC model (simple point charge) [36] was used for water, and the system was submitted to Parrinello-Rahman [36] coupling for temperature and pressure [37,38]. A cut-off of $1.2 \mathrm{~nm}$ was used for coulombic and van der Waals interactions. Partial charges of ACOP were derived from the ChelpG method [38] obtained with Gaussian 09 software [39], using a B3LYP/6-311 G(d) functional and basis set, respectively. The system was first equilibrated for $50 \mathrm{~ns}$. The thermodynamic properties were calculated after a production phase of $100 \mathrm{~ns}$ with a time-step of $2 \mathrm{fs}$ and data collection was undertaken every 0.01 ps. For the ACOP - ionic liquid system, ACOP was immersed in a cubic box with 500 ion pairs. The simulation was carried out in the isothermal-isobaric ensemble (NPT), where the pressure $(\mathrm{P}=1 \mathrm{~atm})$ and the temperature $(\mathrm{T}=298 \mathrm{~K})$ were kept constant by using the Parrinello-Rahman [44] and velocity rescaling [40] schemes. The parameters for the ionic liquid, based on the OPLS-AA force field, were developed by Canongia Lopes and coworkers [41]. Atomic partial charges for $[\mathrm{BMIM}]^{+}$were derived from the electrostatic potential by using the ChelpG method [38]. Periodic boundary conditions and the minimum image convention were applied [35,36]. A cut-off of $1.2 \mathrm{~nm}$ was applied to van der Waals interactions, whereas the electrostatic interactions were treated by the Particle Mesh Ewald method [36,42]. The computational system was first equilibrated for $50 \mathrm{~ns}$. The thermodynamic properties were calculated after a production phase of $100 \mathrm{~ns}$ with a time-step of $2 \mathrm{fs}$ and data collection every $0.01 \mathrm{ps}$.

Monte Carlo simulations using the Adsorption Locator module of the Materials Studio software [42] were performed to study the adsorption of the ion pairs to a modeled nanotube. The ion pair configuration was obtained from the previous molecular dynamics (MD) simulation, and the nanotube was modeled as a 5-walled carbon nanotube functionalized with carboxylic acid on both the external and internal surfaces $(f$ MWCNT), with an external diameter of $72 \mathrm{~nm}$ and length of $42 \mathrm{~nm}$.

Geometry optimization and vibrational frequency calculations were performed using Density functional Theory (DFT) methods as implemented in the Gaussian 09 [39] and Orca 3.0.3 software packages [43] for paracetamol (ACOP), its oxidized form $\left(\mathrm{ACOP}^{+}\right)$and the IL ion pair $[\mathrm{BMIM}]^{+}\left[\mathrm{PF}_{6}\right]^{-}$. DFT was also used to investigate the adsorption of the same species in a large poly aromatic hydrocarbon (PAH) model of the nanotube's surface consisting of 57 carbon and 19 hydrogen capping atoms. This system has been used in all DFT calculations were nanotube adsorption energies were modeled and is referred to simply as graphene model. For the DFT studies, we used the BLYP functional with D3 empirical dispersion corrections and a 6-311 G(d) [43] basis set. For the cases were ACOP and ACOP +1 molecules were adsorbed onto a graphene - IL set, the atomic positions of graphene - IL were kept fixed in their optimized positions. This methodology was previously tested by the group to investigate $\pi$-stacking [44]. 


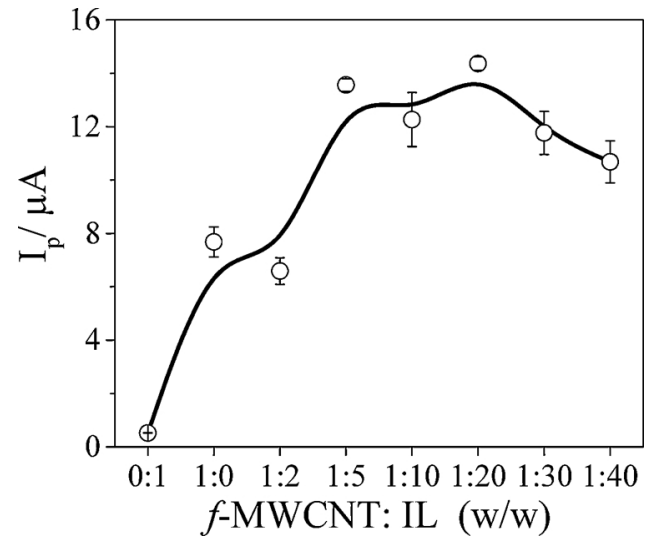

Fig. 1. Values of the oxidation peak current for ACOP $\left(1.19 \times 10^{-5} \mathrm{~mol} \mathrm{~L}^{-1}\right.$ in a Britton-Robinson buffer at $\mathrm{pH}=5.0, f=100 \mathrm{~s}^{-1}, \quad a=50 \mathrm{mV}$ and $\Delta \mathrm{E}_{s}=2 \mathrm{mV}$ ) with the $f$-MWCNT - IL /GCE with different ratios (w/w) of $f$ MWCNT and IL. Error bars are the standard deviation after three replicates.

\section{Results and discussion}

\subsection{Experimental: effect of the ratio of $f$-MWCNTs and $I L$}

The concentration of IL in the dispersion of $f$-MWCNTs was explored using the SWV technique for ACOP sensing with different ratios of GCE/ $f$-MWCNT-IL. The voltammograms were obtained with $f=100 \mathrm{~s}^{-1}$, $a=50 \mathrm{mV}$ and $\Delta \mathrm{E}_{s}=2 \mathrm{mV}$ using a $0.04 \mathrm{~mol} \mathrm{~L}^{-1}$ BR buffer at pH 5.0 in the presence of $1.19 \times 10^{-5} \mathrm{~mol} \mathrm{~L}^{-1}$ ACOP. As shown in Fig. 1, the oxidation peak current of ACOP is very low when there are no $f$ MWCNTs. For a fixed concentration of $f$-MWCNTs $\left(1 \mathrm{mg} \mathrm{mL}^{-1}\right)$ and different concentrations of IL $\left(0.0-20 \mathrm{mg} \mathrm{mL}^{-1}\right)$ the oxidation current dramatically changes, reaching its maximum between a ratio of 1:5 and $1: 20$.

To evaluate whether the diffusion or the adsorption were the ratedetermining step for the electrooxidation mechanism of the ACOP, the scan rate ( $v$ ) was studied (Fig. 2). The relationship between the anodic $\left(I_{p a}\right)$ and cathodic $\left(I_{p c}\right)$ peak currents were found to be linearly dependent on the value of $v$ (Fig. 2B). The regression equations obtained for 10 points are

$\mathrm{I}_{\mathrm{pa}} \quad(\mathrm{A})=2.06 \times 10^{-7} \quad\left( \pm 1.09 \times 10^{-8}\right)+1.27 \times 10^{-8}$

$\left( \pm 3.06 \times 10^{-10}\right) v\left(\mathrm{mV} \mathrm{s}^{-1}\right) ; \mathrm{R}=0.9942$

$\mathrm{I}_{\mathrm{pc}} \quad(\mathrm{A})=3.92 \times 10^{-8} \quad\left( \pm 2.02 \times 10^{-9}\right) \quad-\quad 6.98 \times 10^{-9}$ $\left( \pm 5.65 \times 10^{-11}\right) v\left(\mathrm{mV} \mathrm{s}^{-1}\right) ; \mathrm{R}=0.9994$

Because a linear correlation was obtained between the peak current and the scan rate, we conclude that the redox process is controlled by adsorption rather than the diffusion of ACOP on the electrode surface. This result is in agreement with previous literature reports [45-49].

\subsection{Computational results}

Using the Adsorption Locator module in the Materials Studio software, we have studied the adsorption of different amounts of ion liquid pairs $\left([\mathrm{BMIM}]^{+}\left[\mathrm{PF}_{6}\right]^{-}\right.$) to a functionalized nanotube (f-MWCNT). The adsorption energy ( $E_{a d s}$ ) becomes more negative, and exhibits a linear behavior as the number of ion pairs increases (Fig. 3A). However, when the $E_{a d s}$ values are divided by the quantity of ion pairs being adsorbed, we observed a plateau after 20 ion pairs (Fig. 3B - the insert shows the region where $\mathrm{E}_{\mathrm{ads}}$ increases almost linearly).

We have also performed Monte Carlo simulations for the adsorption of one ACOP molecule on a substrate ( $f$-MWCNT $+x$ IL) using distinct amounts of pre adsorbed ion pairs, with $x=0,2,10,20,50,100$ and 110 ( $x$ being number of IL ion pairs; results are shown in Fig. 4). We analyzed the mean value of $\mathrm{E}_{\text {ads }}$ by considering the 10 most stable
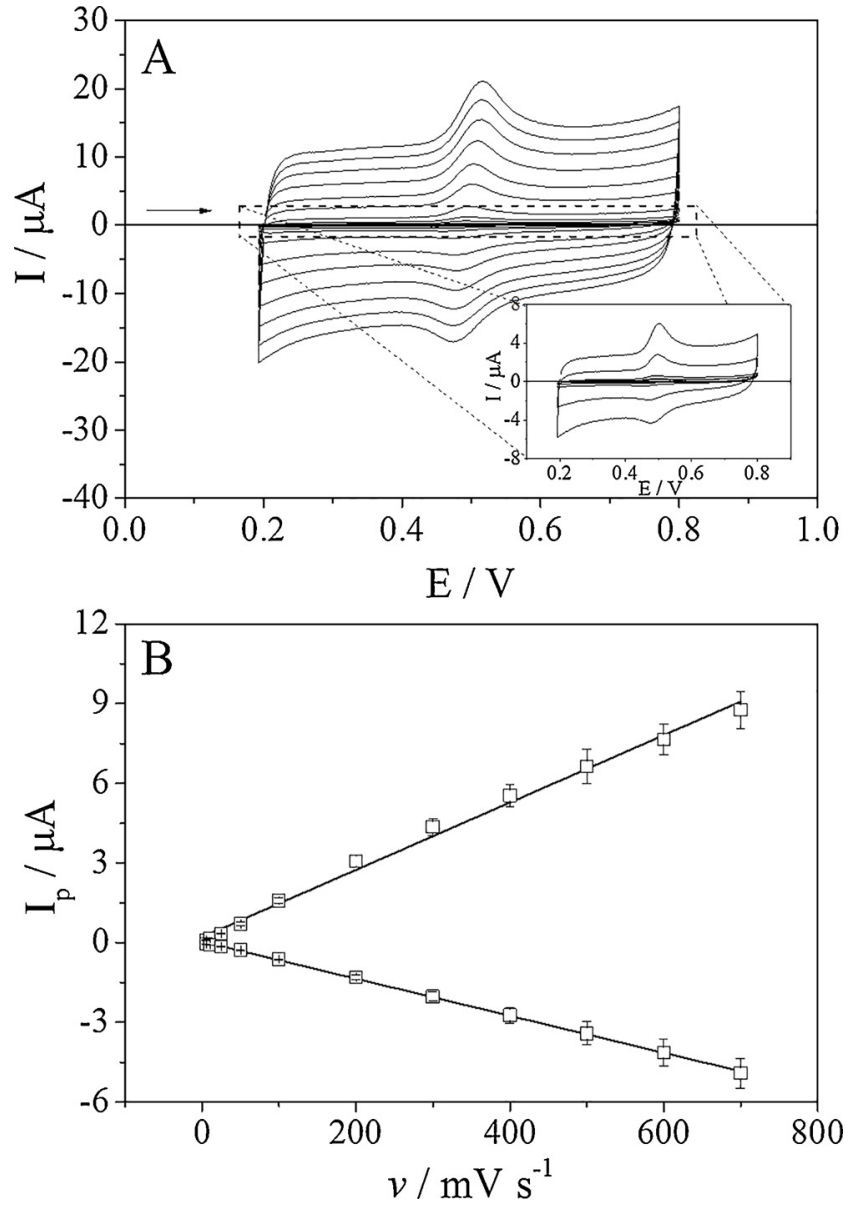

Fig. 2. (A) Cyclic voltammograms of a $1.19 \times 10^{-5} \mathrm{~mol} \mathrm{~L}^{-1}$ ACOP in BR buffer at $\mathrm{pH} 5.0$ on the $f$-MWCNT - IL /GCE (1:5) obtained with different scan rates $\left(5-700 \mathrm{mV} \mathrm{s}^{-1}\right)$. The insert shows scan rates from 5 to $100 \mathrm{mV} \mathrm{s}^{-1}$. (B) Anodic and cathodic peak current versus scan rate. Error bars are the standard deviation of three replicates.

configurations from our simulation results. Comparing the results for Figs. 3 and 4, one notes that the adsorption energy values are similar $\left(-193 v s .-190 \mathrm{kcal}^{\mathrm{mol}}{ }^{-1}\right)$ for ACOP and the initial pairs of IL. For IL pairs, however, adsorption energy quickly decreases reaching a plateau after about 20 pairs. The behavior for ACOP $E_{a d s}$ is distinct: the increase of pre adsorbed IL pair number has little effect on the ACOP $E_{a d s}$, resulting in a nearly constant value after about coverage of 20 IL pairs. Experimentally, we observed that the oxidation process is controlled by adsorption, and, the oxidation peak current increases quickly up to ratio $w / w$ of 1:20 $f$-MWCNT:IL, after which it levels up and decreases.

The picture that emerges from these results is that IL nanotube coverage seems to be more favorable for smaller amount of IL, and that partial small coverage is followed by an experimental increase in current. Interestingly, ACOP adsorption energy does not seem to be much affected by the increase in IL pairs (pre adsorbed), except in the range were about 1-20 IL pairs were considered. In this range, a small decrease in $E_{a d s}$ is observed for 2 and 10 adsorbed pairs, and, for $20 \mathrm{IL}$ pairs, one observes the largest standard deviations from mean values. So, to evaluate specific interactions among the components of this complex system we have carried out simulations using molecular dynamics and quantum mechanical methods using distinct smaller model systems.

Molecular Dynamics simulations were employed to investigate the fundamental interactions between ACOP and the reaction media. We have compared the coulombic interaction term $\left(\mathrm{U}_{\text {coul }}\right)$ and the van der Waals interaction term $\left(\mathrm{U}_{\mathrm{LJ}}\right)$ for ACOP in water to ACOP in ionic liquid. 

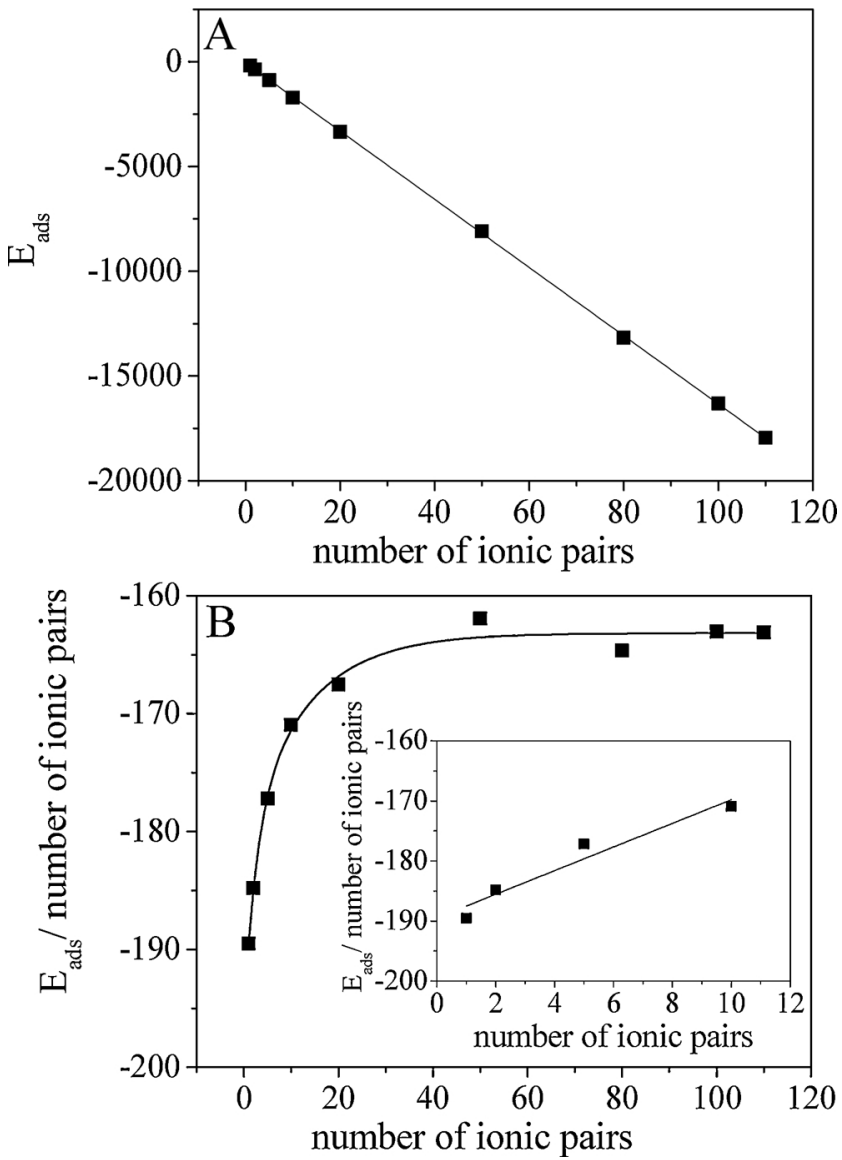

Fig. 3. (A) Adsorption energy in kcal. $\mathrm{mol}^{-1}$ for increasing amounts of ionic liquid on $f$-MWCNT and (B) ratio of adsorption energy $\left(E_{\text {ads }}\right)$ to the number of ionic pairs.

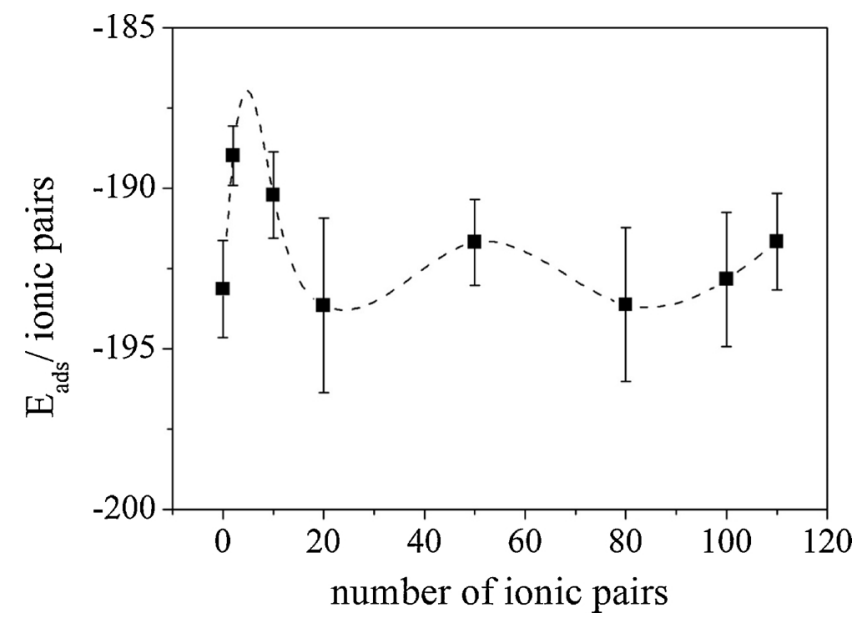

Fig. 4. Adsorption energy in kcal. $\mathrm{mol}^{-1}$ for ACOP on $f$-MWCNT-IL vs. number of pre adsorbed IL pairs.

The results presented in Table 1 show that the interactions between water-ACOP and ionic liquid-ACOP are both attractive; coulombic interactions are prevalent for the water-ACOP interaction, while the van der Waals terms (Lennard Jones) are more significant in ionic liquid.

The radial distribution function (RDF) and an example of mean interactions in solvent (Figs. SM1 and SM2 in Supplementary material), show that these interactions are due to hydrogen bonds formed between the ACOP molecule and water. In IL, there is an attractive coulombic interaction between ACOP and the anion $\left[\mathrm{PF}_{6}\right]^{-}$, whether the $\pi$ -
Table 1

Contribution of each of the energy terms obtained by molecular dynamics simulations (Coulombic $\left(\mathrm{U}_{\mathrm{Coul}}\right)$ and van der Waals - Lennard Jones $\left(\mathrm{U}_{\mathrm{LJ}}\right)$ ) for the system's water - ACOP and ionic liquid (IL) - ACOP.

\begin{tabular}{llll}
\hline & $\mathrm{U}_{\mathrm{LJ}}\left(\mathrm{kcal} . \mathrm{mol}^{-1}\right)$ & $\mathrm{U}_{\mathrm{Coul}}\left(\mathrm{kcal} . \mathrm{mol}^{-1}\right)$ & $\mathrm{U}_{\text {tot }}\left(\mathrm{kcal} . \mathrm{mol}^{-1}\right)$ \\
\hline Water - ACOP & -10.354 & -49.235 & -59.588 \\
Ionic liquid - ACOP & -26.123 & -17.829 & -43.954 \\
[BMIM $^{+}$- ACOP & -19.885 & -2.557 & -22.442 \\
[PF $\left._{6}\right]^{-}$- ACOP & -6.238 & -15.272 & -21.510 \\
\hline
\end{tabular}

interaction between ACOP and $[\mathrm{BMIM}]^{+}$increases the value of $\mathrm{U}_{\mathrm{LJ}}$. From these calculations, we can deduce that the ACOP-water interaction is preferred to ACOP-IL. Therefore, if the nanotube surface is completely covered by IL, ACOP concentration would be higher in aqueous solution.

To further investigate the adsorption/desorption energetics and to gain insight into the enhancement mechanism allowed by the IL, we have carried out simulations using quantum mechanical methods. While we have focused our initial theoretical investigation on the adsorption behavior of neutral ACOP, the desorption of the oxidized species may also have a determining impact on the measured peak current. To investigate the interaction between the nanotube, ionic liquid and the ACOP in different oxidized states, we have performed a set of quantum mechanical calculations using Density Functional Theory, the BLYP-D3 functional and 6-311 G(d) basis set. The previously described graphene small model has been used throughout all quantum calculations.

Four systems were considered: the ACOP/graphene model and the ACOP/IL/graphene model in their neutral and oxidized forms. We have observed from our Monte Carlo simulations that the adsorption of ACOP to the nanotube surface occurs preferentially in the proximity of an IL pair. Therefore, only configurations with ACOP next to an IL pair were considered for the DFT investigation. A stepwise procedure was used to reach the ACOP/LI/graphene model final optimized geometry. First, an initial geometry optimization of one ion pair over the graphene model was performed. Then, the atomic positions of this system were kept fixed and an ACOP molecule (either in its neutral or charged form - $\mathrm{ACOP}^{+}$) was added. Two distinct configurations were considered for the ACOP/LI/graphene model system: one configuration has the ACOP molecule over the IL ion pair and the other, next to it. Adsorption energies $\left(E_{a d s}\right)$, were calculated as the single point energy $\left(E_{T}\right)$ of the whole system (graphene + IL + ACOP or $\mathrm{ACOP}^{+}$) minus the single point energies of graphene $\left(E_{G}\right)$, the ionic pair $\left(E_{I L}\right)$ and ACOP (or $\left.\mathrm{ACOP}^{+}\right)\left(\mathrm{E}_{\mathrm{ACOP}}\right)$, as presented in Eq. (3):

$E_{a d s}=E_{T}-E_{G}-E_{I L}-E_{A C O P}$

The adsorption energies (Table 2) indicated that neutral ACOP prefers to adsorb directly over the graphene surface and next to the ion pair (IL side), but not over it (IL up). In the absence of IL, oxidized ACOP $\left(\mathrm{ACOP}^{+}\right)$adsorbs more strongly to graphene than neutral ACOP.

Table 2

$\mathrm{ACOP}$ and $\mathrm{ACOP}^{+}$adsorption energies $\left(\mathrm{E}_{\mathrm{ads}}\right)$, ratios $\left(\gamma \mathrm{E}_{\mathrm{ads}}=\mathrm{E}_{\mathrm{ads}\left(\mathrm{ACOP}^{+}\right) /}\right)$ $\left.\mathrm{E}_{\mathrm{ads}(\mathrm{ACOP})}\right)$ and energy differences $\left(\Delta \mathrm{E}_{\mathrm{ads}}\right)$ between oxidized $\left(\mathrm{ACOP}^{+}\right)$and neutral forms of ACOP. Results obtained using the graphene model. Results in kcal. $\mathrm{mol}^{-1}$.

\begin{tabular}{|c|c|c|c|c|}
\hline \multirow[b]{2}{*}{ Substrate } & \multicolumn{2}{|l|}{$E_{\text {ads }}$} & \multirow[b]{2}{*}{$\gamma \mathrm{E}_{\mathrm{ads}}$} & \multirow[b]{2}{*}{$\Delta \mathrm{E}_{\text {ads }}$} \\
\hline & ACOP & $\mathrm{ACOP}^{+}$ & & \\
\hline graphene & -95.57 & -301.15 & 3.15 & -205.58 \\
\hline graphene - IL(up*) & -218.70 & -394.87 & 1.81 & -176.17 \\
\hline graphene - IL(side") & -242.07 & -69.58 & 0.29 & 172.50 \\
\hline
\end{tabular}

* up = adsorption occurs over IL; side = adsorption occurs on the nanotube beside one IL pair. 
Table 3

Frontier molecular orbitals energy and the difference (gap) between them (in $\mathrm{eV}$ ) for graphene with and without IL, and with ACOP and ACOP ${ }^{+}$adsorbed.

\begin{tabular}{|c|c|c|c|c|}
\hline & \multicolumn{3}{|c|}{ Molecular orbital $(\mathrm{eV})$} & \multirow{2}{*}{$\begin{array}{l}\text { Atomic charges } \\
\text { on ACOP or } \\
\mathrm{ACOP}^{+}\end{array}$} \\
\hline & HOMO & LUMO & $\begin{array}{l}\text { Gap } \\
\text { (LUMO- } \\
\text { HOMO) }\end{array}$ & \\
\hline Graphene & -3.8066 & -2.8119 & 0.9947 & - \\
\hline Graphene + IL & -3.9586 & -2.8810 & 1.0777 & - \\
\hline ACOP on Graphene & -3.7661 & -2.8345 & 0.9316 & -0.192 \\
\hline $\begin{array}{l}\text { ACOP on (Graphene + IL } \\
\quad \text { - side) }\end{array}$ & -3.9583 & -2.8898 & 1.0686 & -0.018 \\
\hline $\mathrm{ACOP}^{+}$on Graphene & -6.7708 & -6.2873 & 0.4835 & -0.143 \\
\hline $\begin{array}{l}\mathrm{ACOP}^{+} \text {on } \\
\text { (Graphene + IL - } \\
\text { side) }\end{array}$ & -6.4372 & -6.4334 & 0.0038 & 0.141 \\
\hline
\end{tabular}

When IL is present, the opposite occurs: $\mathrm{ACOP}^{+}$adsorption is more likely to occur over the IL (IL up) than on the grapheme model (and much more strongly than next to an IL pair). Our results indicate that the IL role might be two-fold: by facilitating ACOP adsorption (by increasing in adsorption energy) and $\mathrm{ACOP}^{+}$desorption (by decreasing adsorption energy) to the nanotube surface. These trends can be easily identified by observing the large changes in the ratio $\gamma \mathrm{E}_{\mathrm{ads}}=\mathrm{E}_{\mathrm{ACOP}+} /$ $\mathrm{E}_{\mathrm{ACOP}}$ and changes in adsorption energy $\left(\Delta \mathrm{E}_{\mathrm{ads}}\right)$ presented in Table 2. The combined results should have an impact on the ACOP turnaround kinetics leading to the observed current increase.

Table 3 presents the frontier molecular orbital (HOMO and LUMO) energies for the systems studied using the graphene model, and the changes caused by adsorption of IL, ACOP and ACOP ${ }^{+}$. Note that, as we have studied a small graphene model, discrete electronic levels were obtained, and the adsorption of IL has a small effect on them. ACOP adsorption also do not modify the energy of these electronic levels, however, when oxidation occurs $\left(\mathrm{ACOP}^{+}\right.$on graphene or graphene + $\mathrm{IL})$, the whole system is affected and HOMO and LUMO becomes nearly degenerated, resulting in abrupt change in the gap. Results for the charge transfer character of the ACOP/ACOP ${ }^{+}$interaction using Mulliken are shown on Table 3. These charges indicate that graphene donate negative charge to ACOP, but in the presence of IL, this is subdued. Once oxidation occurs, the whole system is affected. When adsorbed to graphene ACOP remains negatively charged, even if the charge is 0.05 smaller. However, when adsorbed to graphene in the presence of IL, ACOP becomes positively charged indicating that the IL favors ACOP oxidation. Fig. 5 displays the main molecular orbitals involved in oxidation process: HOMO for ACOP and LUMO for ACOP ${ }^{+}$. The orbital amplitudes for the LUMO is strongly dependent on the presence of the IL pair. When IL is present, LUMO shows a clear mixture of graphene and ACOP orbitals as opposed to a graphene only LUMO for the case without IL. Therefore, the combined data from electronic structure calculations suggests that the IL helps drive ACOP oxidation.

\section{Conclusions}

Electrochemical studies on the oxidation of acetaminophen (ACOP) promoted by modified nanotubes showed that the ionic liquid, $[\mathrm{BMIM}]^{+}\left[\mathrm{PF}_{6}\right]^{-}$has a strong impact on the observed current used to detect ACOP on a $f$-MWCNTs/GC electrode. Moreover, there is an optimal concentration of ionic liquid $\left([\mathrm{BMIM}]^{+}\left[\mathrm{PF}_{6}\right]^{-}\right.$) that leads to a maximum in the oxidation rate, as measured by the current of the process.

To characterize this phenomenon, we have performed a set of different complementary computational simulations. Monte Carlo studies

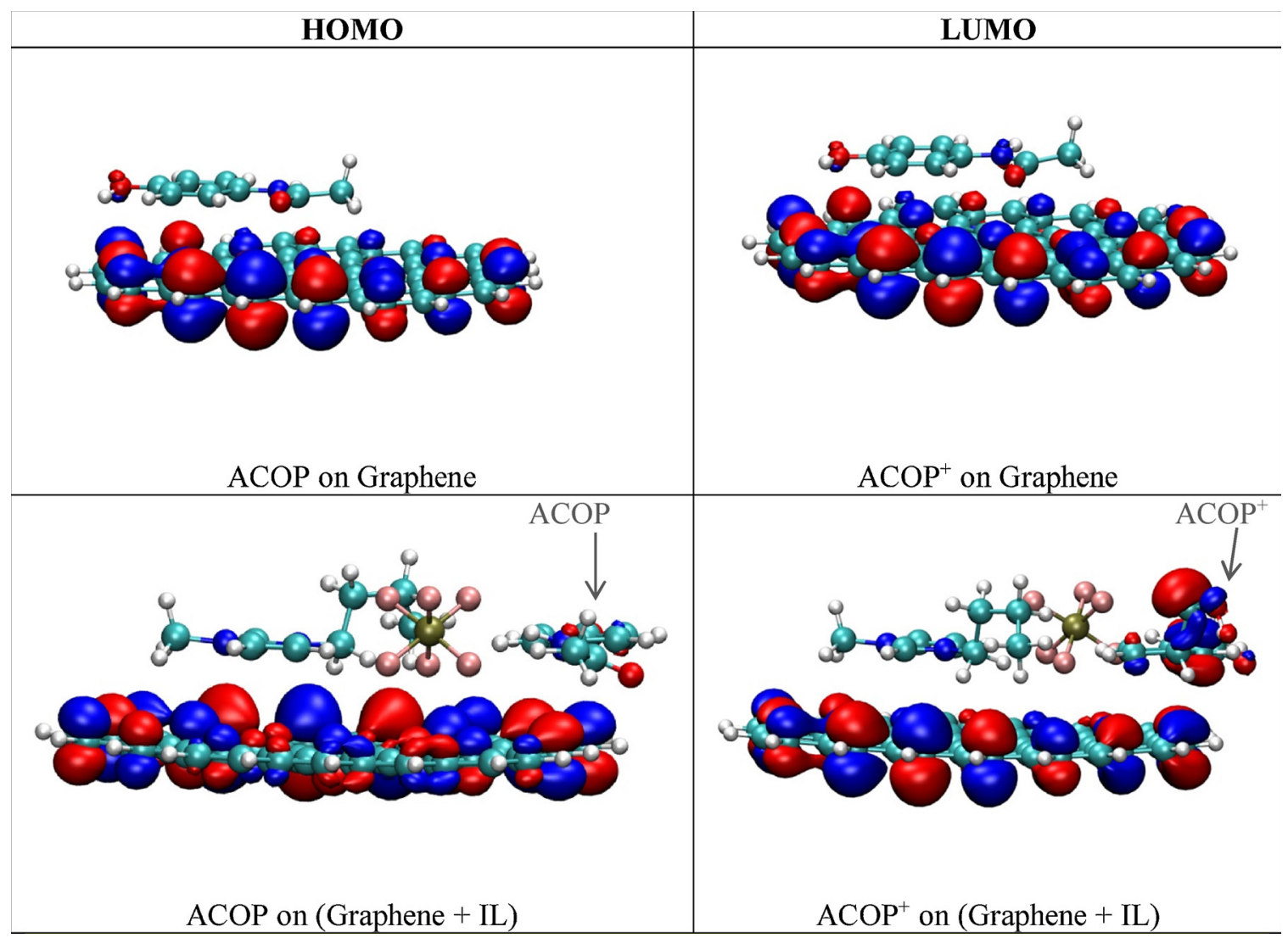

Fig. 5. Molecular orbitals involved on oxidation of ACOP adsorbed on Graphene $+\mathrm{IL}\left([\mathrm{BMIM}]^{+}\left[\mathrm{PF}_{6}\right]^{-}\right.$): HOMO for reduced and LUMO for oxidized species, obtained with BLYP-D3/6-311 G(d). Carbon atoms in cyan, hydrogen atoms in white, oxygen atoms in red, nitrogen atoms in blue, fluorine atoms in pink and phosphorous atom in green (For interpretation of the references to colour in this figure legend, the reader is referred to the web version of this article). 
indicated that the presence of intermediary amounts of ionic liquid on the nanotube increases the adsorption energy, and larger amount of ion pairs furnishes almost constant adsorption energy. From these simulations, we noted that the oxidation process is controlled by adsorption and occurs on the nanotube surface, therefore it is important to have a nanotube surface free of ionic liquid.

To clarify the role of the ionic liquid on the oxidation process, we have performed molecular dynamics and quantum mechanics calculations. Initially, we verified the fundamental interactions between ACOP and the ionic liquid, and ACOP with water, since ACOP is in an aqueous solution before the adsorption. The energy terms from the molecular dynamics calculations suggested that the coulombic interaction between the ACOP and water is prevalent, while for the ionic liquid, the van der Waals term (Lennard Jones) is more significant. This is due to hydrogen bonds formed between ACOP $+1, \mathrm{n}$ the ACOP molecule and the solvent. On the other hand, there is an attractive coulombic interaction between ACOP and the $\left[\mathrm{PF}_{6}\right]^{-}$anion and a $\pi$-type interaction between the ACOP and $[\mathrm{BMIM}]^{+}$, increasing the value of $\mathrm{U}_{\mathrm{LJ}}$. This is caused by the type of interactions among the ACOP molecule and the solvent.

Considering a small graphene-like surface model with an adsorbed ion pair, we have studied the adsorption of ACOP in both neutral and oxidized states. With these calculations, we have shown that IL has a fundamental role on the adsorption and oxidation processes: initially, it increases the adsorption energy of the reduced species; after this, it facilitates oxidation because it affects the charge transfer between ACOP and nanotube (it decreases LUMO-HOMO gap and allows ACOP to be positively charged); and, finally, it reduces the adsorption energy of $\mathrm{ACOP}^{+}$, facilitating the overall turnover and resulting in an increased oxidation speed.

Therefore, the proposed sensor, constructed from the carbon nanotubes and $[\mathrm{BMIM}]^{+}\left[\mathrm{PF}_{6}\right]^{-}$at ratio between 1:5 and 1:20, may be suitable for ACOP detection. Those ratios are selected because of the synergistic effect between nanotube surface and IL: ACOP adsorption may occur on the nanotube surface, but oxidation and desorption process of $\mathrm{ACOP}^{+}$(and consequently the clearance of the area for new adsorptions) depends on the presence of appropriate quantities of IL.

\section{Acknowledgments}

The authors wish to thank the Brazilian research funding institutions Conselho Nacional de Desenvolvimento Científico e Tecnológico (CNPq) and Fundação de Amparo à Pesquisa do Estado de São Paulo (FAPESP) for their financial support (CNPq proc. 400223/2014-7, 303596/2014-7, 306177/2016-1，302801/2014-6, 154089/2016-8, 573925/2008-9 and 573548/2008-0; FAPESP 2017/23416-9) and CAPES (Funcap - 2133/2012/proc. 23038.007973/2012-90), PRONEM/FUNCAP/CNPq (PNE-0112-00048.01.00/16), PRONEX/ FUNCAP (proc. PR2-0101-00030.01.00/15). This study was financed in part by the Coordenação de Aperfeiçoamento de Pessoal de Nível Superior - Brasil (CAPES) - Finance Code 00. Camila P. Sousa thanks CAPES- PNPD for her grant.

\section{Appendix A. Supplementary data}

Supplementary material related to this article can be found, in the online version, at doi:https://doi.org/10.1016/j.snb.2018.09.017.

\section{References}

[1] M.R. Akanda, M. Sohail, M.A. Aziz, A.N. Kawde, Recent advances in nanomaterialmodified pencil graphite electrodes for electroanalysis, Electroanalysis 28 (3) (2016) 408-424.

[2] D. Bellido-Milla, et al., Recent advances in graphite powder-based electrodes, Anal. Bioanal. Chem. 405 (11) (2013) 3525-3539.

[3] K. Kalcher, I. Svancara, M. Buzuk, K. Vytras, A. Walcarius, Electrochemical sensors and biosensors based on heterogeneous carbon materials, Monatshefte für Chemie
Chem. Mon. 140 (8) (2009) 861-889.

[4] D. Sharp, R. Burkitt, Carbon materials for analytical electrochemistry: printed carbon materials and composites, Mater. Technol. 30 (sup. 7) (2015) B155-B162.

[5] A.J.S. Ahammad, J.-J. Lee, M.A. Rahman, Electrochemical sensors based on carbon nanotubes, Sensors 9 (4) (2009) 2289-2319.

[6] A. Brotons, F.J. Vidal-Iglesias, J. Solla-Gullón, J. Iniesta, Carbon materials for the electrooxidation of nucleobases, nucleosides and nucleotides toward cytosine methylation detection: a review, Anal. Methods 8 (4) (2016) 702-715.

[7] D.R. Kauffman, A. Star, Graphene versus carbon nanotubes for chemical sensor and fuel cell applications, Analyst 135 (11) (2010) 2790-2797.

[8] A.T. Lawal, Synthesis and utilization of carbon nanotubes for fabrication of elec trochemical biosensors, Mater. Res. Bull. 73 (2016) 308-350.

[9] F. Li, S. Gan, D. Han, L. Niu, Graphene-based nanohybrids for advanced electrochemical sensing, Electroanalysis 27 (9) (2015) 2098-2115.

[10] R. Honghong, X. Zhonghua, W. Xuemei, Z. Guohu, H. Huihui, W. Hui, Electrochemical sensors based on electrochemically reduced graphene oxide, Progress Chem. 28 (2/3) (2016) 337-352.

[11] K.R. Ratinac, W. Yang, J.J. Gooding, P. Thordarson, F. Braet, Graphene and related materials in electrochemical sensing, Electroanalysis 23 (4) (2011) 803-826.

[12] R.K. Singh, R. Kumar, D.P. Singh, Graphene oxide: strategies for synthesis, reduction and frontier applications, RSC Adv. 6 (69) (2016) 64993-65011.

[13] P. Yáñez-Sedeño, J.M. Pingarrón, J. Riu, F.X. Rius, Electrochemical sensing based on carbon nanotubes, TrAC - Trends Anal. Chem. 29 (9) (2010) 939-953.

[14] M. Noked, A. Soffer, D. Arubach, The electrochemistry of activated carbonaceous materials: past, present, and future, J. Solid State Electrochem 15 (7-8) (2011) 1563-1578.

[15] K. Balasubramanian, M. Burghard, Chemically functionalized carbon nanotubes, Small 1 (2) (2005) 180-192.

[16] M. Shim, N.W.S. Kam, R.J. Chen, Y. Li, H. Dai, Functionalization of carbon nanotubes for biocompatibility and biomolecular recognition, Nano Lett. 2 (4) (2002) 285-288

[17] M. Opallo, A. Lesniewski, A review on electrodes modified with ionic liquids, J. Electroanal. Chem. 656 (1-2) (2011) 2-16.

[18] A. Abo-Hamad, M.A. Alsaadi, M. Hayyan, I. Juneidi, M.A. Hashim, Ionic liquidcarbon nanomaterial hybrids for electrochemical sensor applications: a review, Electrochim. Acta 193 (2016) 321-343.

[19] A.-M. Gurban, L. Rotariu, M. Baibarac, I. Baltog, C. Bala, Sensitive detection of endocrine disrupters using ionic liquid-single walled carbon nanotubes modified screen-printed based biosensors, Talanta 85 (4) (2011) 2007-2013.

[20] Y. Li, et al., Non-enzymatic sensing of uric acid using a carbon nanotube ionic-liquid paste electrode modified with poly( $\beta$-cyclodextrin), Microchim. Acta 182 (11-12) (2015) 877-1884

[21] B. Liu, M. Wang, B. Xiao, Application of carbon nanotube-ionic liquid-epinephrine composite gel modified electrode as a sensor for glutathione, J. Electroanal. Chem. 757 (2015) 198-202.

[22] M.C. Kroon, W. Buijs, C.J. Peters, G.-J. Witkamp, Decomposition of ionic liquids in electrochemical processing, Green Chem. 8 (3) (2006) 241-245.

[23] R. Singh, J. Monk, F.R. Hung, A computational study of the behavior of the ionic liquid [BMIM +] [PF 6 - ] confined inside multiwalled carbon nanotubes, J. Phys. Chem. C (2010) 15478-15485.

[24] Y. Shim, H.J. Kim, Solvation of carbon nanotubes in a room-temperature ionic liquid, ACS Nano 3 (7) (2009) 1693-1702.

[25] H. Roohi, S. Khyrkhah, Green chemical functionalization of single-wall carbon nanotube with methylimidazolium dicyanamid ionic liquid: a first principle computational exploration, J. Mol. Liq. 211 (2015) 498-505.

[26] P. a Gauden, A.P. Terzyk, R. Pieńkowski, S. Furmaniak, R.P. Wesołowski, P. Kowalczyk, Molecular dynamics of zigzag single walled carbon nanotube immersion in water, Phys. Chem. Chem. Phys. 13 (13) (2011) 5621-5629.

[27] G. García, M. Atilhan, S. Aparicio, Theoretical study of renewable ionic liquids in the pure State and with graphene and carbon nanotubes, J. Phys. Chem. B 119 (37) (2015) 12224-12237.

[28] L.M. Alarcón, D.C. Malaspina, E.P. Schulz, M.A. Frechero, G.A. Appignanesi, Structure and orientation of water molecules at model hydrophobic surfaces with curvature: from graphene sheets to carbon nanotubes and fullerenes, Chem. Phys. $388(1-3)(2011)$ 47-56.

[29] A. Merkoçi, Carbon nanotubes in analytical sciences, Microchim. Acta 152 (3-4) (2006) 157-174 SPEC. ISS.

[30] S. Yilmaz, Sensitive voltammetric determination of paracetamol on poly(4-aminobenzene sulfonic acid) modified glassy carbon electrode, Int. J. Electrochem. Sci. 11 (2016) 6244-6255

[31] Z. Wang, M.D. Shirley, S.T. Meikle, R.L.D. Whitby, S.V. Mikhalovsky, The surface acidity of acid oxidised multi-walled carbon nanotubes and the influence of in-situ generated fulvic acids on their stability in aqueous dispersions, Carbon N. Y. 47 (1) (2009) 73-79.

[32] K.A. Wepasnick, B.A. Smith, K.E. Schrote, H.K. Wilson, S.R. Diegelmann, D.H. Fairbrother, Surface and structural characterization of multi-walled carbon nanotubes following different oxidative treatments, Carbon N. Y. 49 (1) (2011) 24-36.

[33] V. Mirceski, S. Komorsky-Lovric, M. Lovric, Square-Wave Voltammetry: Theory and Application, Springer Science \& Business Media, Berlin Hildelberg, 2007.

[34] H.J.C. Berendsen, D. van der Spoel, R. van Drunen, Comput. Phys. Commun. 91 (1995) 43-56

[35] M.P. Allen, D.J. Tildesley, Computer Simulation of Liquids, 2nd ed., Pearson Education Limited, 20171996.

[36] A. Leach, Molecular Modelling: Principles and Applications, (2001).

[37] H.J.C. Berendsen, J.P.M. Postma, J. van Gunsteren, W.F. Hermans, Interaction 
models of water in relation to protein hydration, Intermolecular Forces: Proceedings of the Fourteenth Jerusalem Symposium on Quantum Chemistry and Biochemistry, B (1981) 331-342.

[38] H. Bekker, et al., Gromacs: a parallel computer for molecular dynamics simulations, in: R.A. deGroot, J. Nadrchal (Eds.), Physics Computing 92 (1993).

[39] M.J. Frisch, et al., Gaussian 09 Revision D.01, Gaussian Inc., Wallingford CT, 2013.

[40] G. Bussi, D. Donadio, M. Parrinello, Canonical sampling through velocity rescaling, J. Chem. Phys. 126 (1) (2007).

[41] J.N. Canongia Lopes, J. Deschamps, A.A.H. Pádua, Modeling ionic liquids using a systematic all-atom force Field, J. Phys. Chem. B 108 (30) (2004) 11250.

[42] T. Darden, D. York, L. Pedersen, Particle mesh Ewald: an N.log(N) method for Ewald sums in large systems, J. Chem. Phys. 98 (12) (1993) 10089-10092.

[43] F. Neese, The ORCA Program System vol. 2, (2012) pp. 73-78.

[44] F. Bettanin, T.A. de Carvalho Fontinelles, C.D. Maciel, L.G. Dias, M.D. CoutinhoNeto, P. Homem-de-Mello, Aggregation of photosensitizers: the role of dispersion and solvation on dimer formation energetics, Theor. Chem. Acc. 134 (12) (2015) $1-11$.

[45] X. Kang, J. Wang, H. Wu, J. Liu, I.A. Aksay, Y. Lin, A graphene-based electrochemical sensor for sensitive detection of paracetamol, Talanta 81 (3) (2010) 754-759.

[46] D. Lu, Y. Zhang, L. Wang, S. Lin, C. Wang, X. Chen, Sensitive detection of acetaminophen based on $\mathrm{Fe} 3 \mathrm{O} 4$ nanoparticles-coated poly(diallyldimethylammonium chloride)-functionalized graphene nanocomposite film, Talanta 88 (2012) 181-186.

[47] T.-Y. Huang, C.-W. Kung, H.-Y. Wei, K.M. Boopathi, C.-W. Chu, K.-C. Ho, A high performance electrochemical sensor for acetaminophen based on a rGO-PEDOT nanotube composite modified electrode, J. Mater. Chem. A 2 (20) (2014) 7229-7237.

[48] J. Li, et al., High-sensitivity paracetamol sensor based on Pd/graphene oxide nanocomposite as an enhanced electrochemical sensing platform, Biosens. Bioelectron. 54 (2014) 468-475.

[49] J.I. Gowda, D.G. Gunjiganvi, N.B. Sunagar, M.N. Bhat, S.T. Nandibewoor, MWCNT-CTAB modified glassy carbon electrode as a sensor for the determination of paracetamol, RSC Adv. 5 (61) (2015) 49045-49053.

Michele A. Salvador is Bachelor in Physics, and received her DSc in Nanosciences and Advanced Materials in 2014 from Federal University of ABC (Brazil). Currently, she is postdoctoral fellow at Federal University of $A B C$ (Brazil). Her research interest is mainly the use of computational tools to investigate properties of nanostructured and molecular systems, using classical and quantum methodologies as DFT, Molecular Dynamics and Monte Carlo.

Camila P. Sousa received her PhD in Nanosciences and Advanced Materials in 2014from Federal University of ABC (Brazil). Currently, she is postdoctoral at Analytical Chemistry and Physical Chemistry Department, Federal University of Ceará (Brazil). Her research interest is mainly in chemically modified electrodes and electroanalysis.

Cleiton D. Maciel holds a Bachelor's Degree in Physics from the Institute of Physics of the University of São Paulo (2007) and a Ph.D. in Nanosciences and Advanced Materials from the Federal University of ABC (2013). He is currently Professor at the Federal Institute of Education, Science and Technology of São Paulo. Performs postdoctoral studies at the Postgraduate Program in Science and Technology / Chemistry of the Federal University of $\mathrm{ABC}$, whose research theme is the theoretical study of photosensitizing compounds with potential application in Photodynamic Therapy through computational simulations. He has experience in the areas of Physics and Chemistry of Condensed Matter with emphasis in Physical Chemistry of Materials, Theoretical and Computational Chemistry, working in the following subjects: Molecular Dynamics, Condensed Matter Thermodynamics, QM / MM Hybrid Methods, Spectroscopy, Electronic Structure.

Rayane N. Gomes is currently an undergrad student at Federal University of Ceará (Brazil). Her research interest is mainly in electrochemistry and sensors.

Simone Morais is a professor in Chemical Engineering Department, Instituto Superior de Engenharia do Porto. She received her doctor degree (Chemical Engineering) from Faculdade de Engenharia da Universidade do Porto in 1998, respectively. Her current research interests include chemically modified electrodes, bioelectroanalysis, preparation and application of nanofunctional materials and new methodologies for environmental pollutant analysis.

Pedro de Lima-Neto is a full professor in Analytical Chemistry and Physical Chemistry Department, Federal University of Ceará (Brazil). He received his master degree (Physics Chemistry) and doctor degree (Physics Chemistry) from São Paulo University (Brazil) in 1991 and in 1996, respectively. His current research interests include metals and alloys electrodeposition, corrosion studies, chemically modified electrodes and electroanalysis.

Maurício D. Coutinho-Neto holds a bachelor's degree in chemistry from the Federal University of Pernambuco (1991), a Ph.D. in Chemistry from the University of Florida (2001) and a postdoctoral fellowship from the Technische Universität München (2004) and the Ecole Polytechnique Fédérale de Lausanne (2006). He is currently an adjunct professor at ABC Federal University. Has experience in the area of computational simulation in chemical and biological systems, working mainly in the following subjects: molecular dynamics, ab initio reactive dynamics, dispersion interactions, theoretical description of REDOX processes.
Adriana Nunes Correia is a professor in Analytical Chemistry and Physical Chemistry Department, Federal University of Ceará (Brazil). She received her master degree (Physics Chemistry) and doctor degree (Physics Chemistry) from São Paulo University (Brazil) in 1994 and in 1998, respectively. Her current research interests include chemically modified electrodes, bioelectroanalysis, preparation and application of nanofunctional materials and new methodologies for environmental pollutant analysis.

Paula Homem-de-Mello is associate professor at Federal University of ABC (Brazil). She received her master degree (Physics Chemistry) and doctor degree (Physics Chemistry) from São Paulo University (Brazil) in 2002 and in 2006, respectively. Her current research interests include Computer Simulation, acting on the following topics: dyes, metal complexes, redox reactions, biomolecules, drugs, nanostructured materials, DFT and PCM. 